\title{
Eight-Year Survival Following Left Hepatic Trisectionectomy With Combined Hepatic Artery And Portal Vein Resection For Advanced Perihilar Cholangiocarcinoma
}

Haitham Triki ( $\nabla$ haitham.triki@hotmail.com )

Rennes 1 University: Universite de Rennes 1 https://orcid.org/0000-0002-9915-9219

Heithem Jeddou

Rennes 1 University: Universite de Rennes 1

Stylianos Tzedakis

Rennes 1 University: Universite de Rennes 1

Dihia Belabbas

Rennes 1 University: Universite de Rennes 1

Solène Florence Kammerer-Jacquet

Rennes 1 University: Universite de Rennes 1

Laurent Sulpice

Rennes 1 University: Universite de Rennes 1

Karim Boudjema

Rennes 1 University: Universite de Rennes 1

\section{Case Report}

Keywords: biliary malignancy, extended hepatectomy, vascular resection

Posted Date: June 9th, 2021

DOl: https://doi.org/10.21203/rs.3.rs-533168/v1

License: (c) (1) This work is licensed under a Creative Commons Attribution 4.0 International License. Read Full License 


\section{Abstract}

We report the case of a patient with exceptional survival over 8 years after left trisectionectomy combined with portal vein and hepatic artery resection and reconstruction for advanced perihilar cholangiocarcinoma. Such extended hepatectomy with vascular resection is the only way to obtain free tumor margin. It can be performed with acceptable morbidity and mortality and it is the only hope to prolong survival.

\section{Introduction}

The main goal of surgery for perihilar cholangiocarcinoma $(\mathrm{PHC})$ is to achieve complete resection with negative margins. Due to the intimate anatomical relationship between the biliary confluence and the vascular inflow at the hepatic hilum, the tumor can rapidly invade the portal vein and/or the hepatic artery. In some patients with advanced disease, extended hepatectomies with vascular resection and reconstruction are required to reach tumor-free margins. In the hands of an experienced surgeon, this surgery can be performed with acceptable morbidity and mortality [1]; however, oncological results remain controversial.

Herein, we report the case of a patient with exceptional survival over 8 years after left trisectionectomy combined with portal vein and hepatic artery resection for advanced PHC.

\section{Case Report}

A 65-year-old woman with no previous medical conditions presented with jaundice. Biological tests showed severe cholestasis. Abdominal ultrasound revealed intrahepatic bile duct dilatation and a flat gallbladder. Abdominal computed tomography (CT) scan and magnetic resonance cholangiopancreatography (MRCP) showed a $30 \mathrm{~mm}$ tumor originating from the biliary convergence suggestive of perihilar cholangiocarcinoma (Fig. 1). The tumor was mainly left sided involving the confluence of the left lateral sectional bile ducts (B2, 3) and left medial sectional branches (B4). On the right side, the tumor reached the confluence of the right anterior and posterior sectoral branches. The confluence of segment 6 (B6) and segment 7 (B7) bile ducts was free from tumor invasion. Concerning vascular involvement, there was an anatomical variation with a common hepatic artery emerging from the superior mesenteric artery. The left hepatic artery was totally included in the tumor. The right hepatic artery had an intratumoral course over a length of $10 \mathrm{~mm}$ with slight stenosis. The bifurcation between the right anterior and right posterior sectoral arteries was tumor-free. The portal vein presented a modal distribution; its left branch was invaded by the tumor, resulting in total left hepatic atrophy. The tumor extended to the portal vein bifurcation, but the right axis remained patent. The right anterior and right posterior portal bifurcation remained tumor free. Chest CT showed no pulmonary extension.

This PHC was classified as Bismuth IV and type X of Rennes [2]. It was decided to perform left trisectionectomy, caudate lobectomy and resection of the main bile duct with simultaneous resection of 
the portal vein and right hepatic artery. A percutaneous transhepatic biliary drain was inserted into the right posterior sectoral bile duct for external drainage. Two weeks later, jaundice disappeared, and the bilirubin level was $25 \mu \mathrm{mol} / / \mathrm{l}$. There was no sign of infection, and the nutritional status of the patient was adequate. The future remnant liver volume (right posterior sector) was $40 \%$ of the total liver volume.

Intraoperative exploration using visualization, palpation and intraoperative color-Doppler ultrasound (IOUS) confirmed the preoperative findings. Liver parenchyma transection was performed under intermittent pedicle and vena cava clamping of $26 \mathrm{~min}$ in total. The hepatic artery was reconstructed using an autologous saphenous vein graft interposed between the right posterior sectoral artery and the common hepatic artery. The reconstruction lasted $32 \mathrm{~min}$. Portal vein reconstruction was performed with end-to-end anastomosis between the main portal vein and the right posterior sectoral vein, while the liver remained arterialized. IOUS confirmed the patency of the reconstructed vessels and the adequate vascularization of the right posterior segments. Intraoperative frozen section analysis of B6, 7 confluence revealed that margins were positive, imposing a more proximal section. B6 and B7 were then separated. Biliary reconstruction using the Roux-en-Y jejunum limb was performed over the two separate segmental bile ducts. Transanastomotic drainage of both ducts was performed according to Volker's technique.

The operative time and intraoperative blood loss were $365 \mathrm{~min}$ and $800 \mathrm{ml}$, respectively. Postoperative outcomes were mainly marked by the occurrence of bilioma treated with cutaneous drainage and antibiotics. The patient was discharged on postoperative day 32 .

Macroscopic analysis of the specimen showed a sclerosing tumor that adhered to both the portal vein and right hepatic artery. Histological examination showed moderately differentiated biliary adenocarcinoma invading surrounding adipose tissue. However, no microscopic invasion of the portal vein or of the right hepatic artery was revealed. The distance between the tumor and the wall of the portal vein was $0.41 \mathrm{~mm}$ (Fig. 2). Perineural invasion was present. Three lymph nodes were found in the lymphadenectomy specimen and were tumor free. All margins were negative. Eventually, the tumor was classified as pT2aNOMO, R0, or stage II according to the TNM staging of perihilar bile duct tumors (AJCC 8th edition).

The patient was included in the French phase III randomized trial PRODIGE 12 and received 12 cycles of adjuvant gemcitabine-oxaliplatin (GEMOX). She is now healthy with no sign of recurrence 8 years after surgery.

\section{Discussion}

PHC type $X$, according to the Rennes classification, is defined by the invasion of the left lateral sectional bile duct confluence (B2, 3). In PHC type X, the left lobe cannot be preserved. Since the biliary confluence of the right and left hemi livers is also invaded by the tumor, the right hepatic artery is commonly invaded. Indeed, the biliary confluence is shifted on the right side of the hepatic hilum and at a close vicinity of the right hepatic artery [2]. Type X PHC includes Type IIIb and type IV of the Bismuth-Corlette classification. 
Hepatic arterial involvement is traditionally considered a contraindication to resection. For such advanced PHC, major hepatectomy combined with arterial and portal resection remains the only possibility for obtaining negative margins, although in some earlier studies, it showed higher mortality rates compared to hepatectomy without vascular resection [3-4]. Currently, experience and advances in complex hepatic and vascular surgical techniques have led to more aggressive approaches with acceptable outcomes $[1,3,5]$. Nagino et al. reported a large series of 50 combined arterial and portal resections with a perioperative mortality of $2 \%$. In this study, the rate of complications related to vascular reconstruction was low [1]. In another study, concomitant hepatic artery resection showed no significant differences in the overall incidence of postoperative complications compared to standard resection, except for postoperative liver abscess [5].

The fibrotic peritumoral response leads to the development of fibrous tissue that may or may not contain islets of tumor cells. Therefore, some tumors seem to macroscopically invade the portal vein or hepatic artery without any microscopic involvement. Nagino et al. found microscopic invasion of the resected portal vein and hepatic artery in $88 \%$ and $54 \%$, respectively [1]. At the time of resection, tumor separation from the vessels should not be attempted to avoid the risk of R1 resection and vascular injury. For such advanced tumors, the "no touch" technique is recommended to increase the likelihood of R0 resection.

Vascular invasion is related to more advanced disease and is known as a poor prognostic factor. However, compared to unresectable tumors or resection with positive margins, combined portal vein and hepatic artery resection offers improved survival when feasible [1,3-5].

\section{Conclusion}

Our case report demonstrates that extended hepatectomy with combined portal vein and hepatic artery resection is feasible and that such extended surgery is the only way to obtain an R0 margin and eventually prolong survival. Innovations and advances in surgical techniques should continue to improve the resectability and long-term survival for advanced PHCs.

\section{Declarations}

Funding: None.

Conflict of interest/Competing interests: The authors have no conflicts of interest to declare.

Ethics approval: For this type of study formal consent is not required.

Consent to participate: Not applicable.

Consent for publication: The patient has consented to the submission of the case report for submission to the journal. 
Availability of data and material: The authors confirm that the data supporting the findings of this study are available within the article and its supplementary materials.

Code availability: Not applicable

Authors' contributions: All authors contributed to the study conception and design. Material preparation, data collection and analysis were performed by Haitham Triki and Heithem Jeddou. The first draft of the manuscript was written by Haitham Triki and all authors commented on previous versions of the manuscript. All authors read and approved the final manuscript.

\section{References}

1. Nagino $M$, Nimura $Y$, Nishio $H$, et al. Hepatectomy with simultaneous resection of the portal vein and hepatic artery for advanced perihilar cholangiocarcinoma: an audit of 50 consecutive cases. Ann Surg 2010;252:115-23.

2. Boudjema K, Sulpice L, Garnier S, et al. A simple system to predict perihilar cholangiocarcinoma resectability. J Gastrointest Surg 2013;17:1247-1256.

3. Higuchi R, Yazawa T, Uemura S, et al. Surgical Outcomes for Perihilar Cholangiocarcinoma with Vascular Invasion. J Gastrointest Surg 2019;23:1443-1453.

4. Schimizzi GV, Jin LX, Davidson JT, et al. Outcomes after vascular resection during curative-intent resection for hilar cholangiocarcinoma: a multi-institution study from the US extrahepatic biliary malignancy consortium. HPB (Oxford) 2018;20:332-339.

5. Noji T, Tsuchikawa T, Okamura K, et al. Concomitant hepatic artery resection for advanced perihilar cholangiocarcinoma: a case-control study with propensity score matching. J Hepatobiliary Pancreat Sci. 2016 Jul;23:442-448.

\section{Figures}



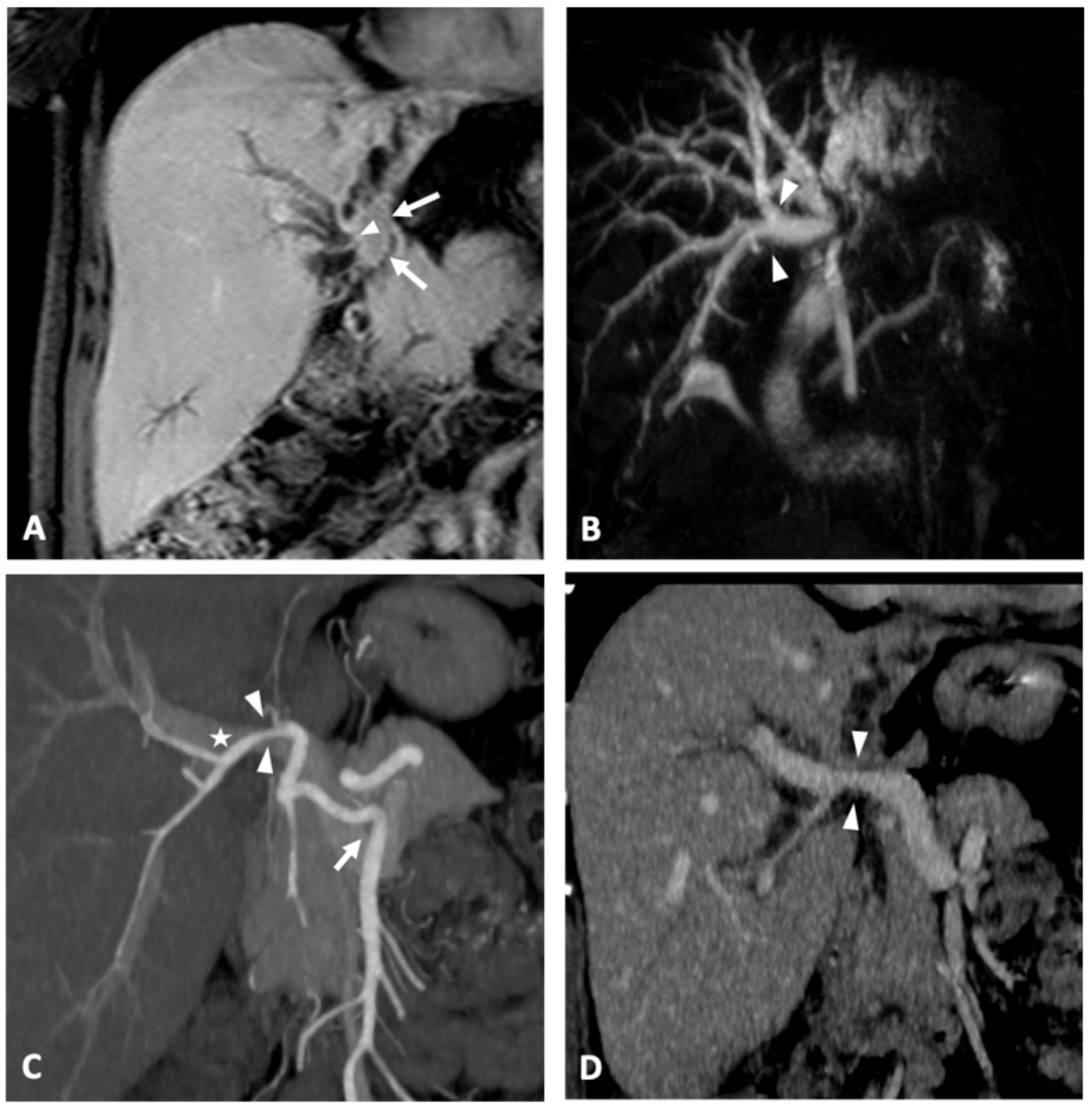

\section{Figure 1}

Preoperative images of perihilar cholangiocarcinoma Bismuth IV and type X of Rennes. A. Magnetic resonance cholangiopancreatography (MRCP) in coronal plane T1-weighted sequence with gadolinium injection at 3 min shows tumoral extension on the left side beyond the confluence of the left lateral sectional bile ducts (B2-3) (white arrows). The tumor reaches the roof of the confluence between the right anterior and posterior sectoral branches (head arrow). B. 3D-MRCP sequence, MIP reconstruction (Maximum Intensity Projection) demonstrates that the confluence of the right posterior inferior branch 
(B6) and the right posterior superior branch (B7) is free (head arrows). C. Computed tomography (CT) scan in coronal plane during arterial phase with MIP reconstruction shows an anatomical variant with common hepatic artery emerging from the superior mesenteric artery (white arrow) and the encasement of right hepatic artery (head arrows). The bifurcation between the right anterior and right posterior sectoral arteries is clear (star). D. CT scan in coronal plane during portal phase reveals tumor invasion of the portal vein with short stenosis before the bifurcation between right anterior and right posterior sectoral portal veins (head arrows).
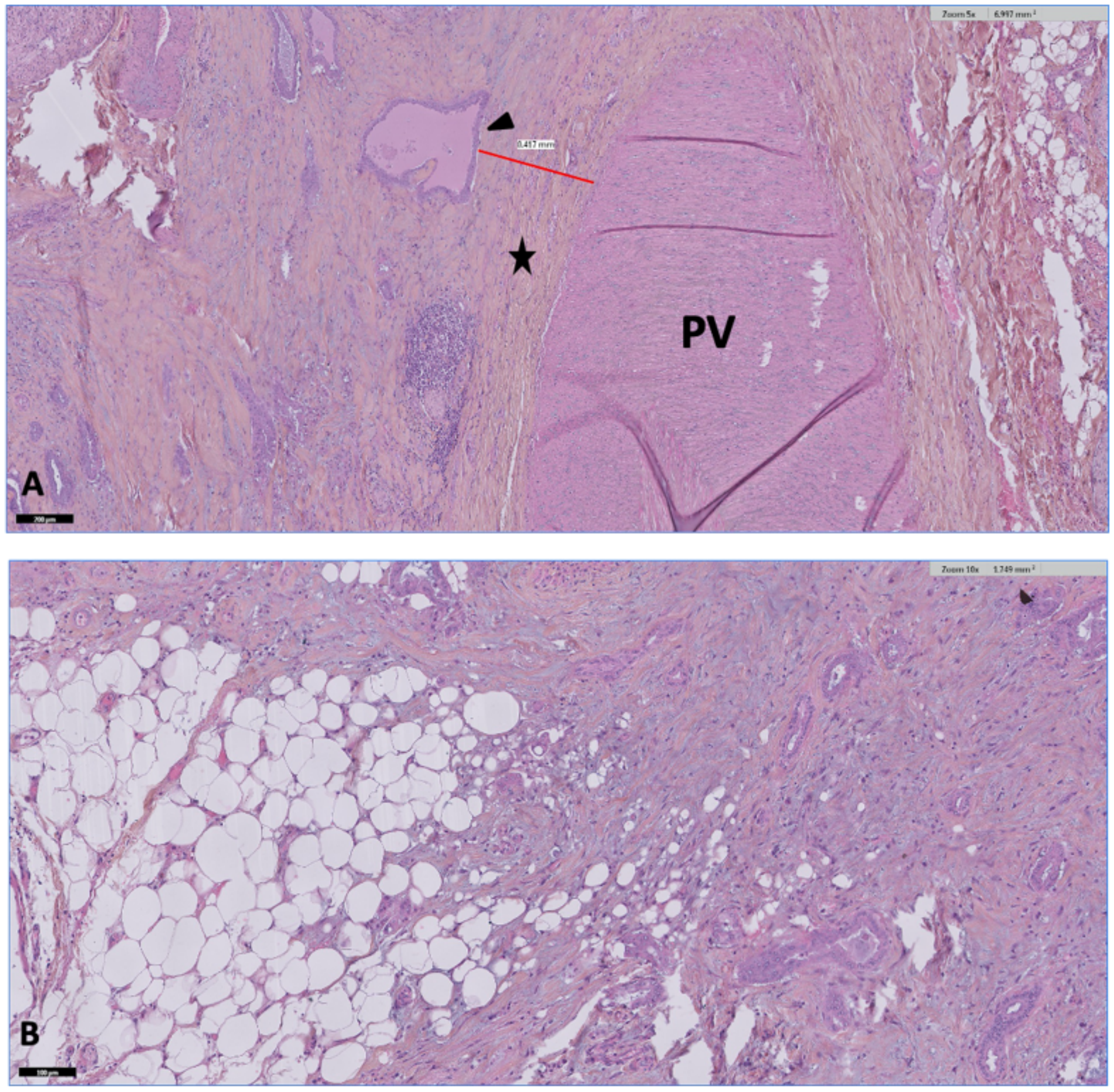

\section{Figure 2}


Histologic findings: A. The portal vein (PV) was not involved histologically. The distance between the wall of the portal vein and the tumor cells (head arrows) was $0,417 \mathrm{~mm}$. Fibrous tissue all around the portal vein (star). B. Tumoral involvement to surrounding adipose tissue. 